\section{A Chemist's Guide to Valence Bond Theory}

by Sason S. Shaik and Phillippe C. Hiberty, John Wiley \& Sons, Hoboken, NJ, USA, 2007, pp 316, ISBN 978-0-470-03735-5, Price: USD 105.00, GBP 70.50

This book has been written as an introduction to modern valence bond theory and new developments for its computational implementation. It is particularly good as a reference source as it explains many commonly used terms and equations in a clear and concise manner. The author covers a wide range of aspects of the theory, from the basic principles to a discussion of its various applications in a wide range of chemical problems. Each chapter is divided into subsections with a short introduction to the chapter followed by definitions, terminology, theory and exercises/answers.
The first chapter briefly summarizes the story of VB theory, placing it into a modern context and setting the scene for the following chapters. Readers should not be put off by the large number of equations and output in the opening chapters as these are very well thought out and a great introduction to the terminology used in later chapters. Chapter 2 continues with a general introduction to VB theory. Chapter 3 gives a more in-depth look at the theory, covering writing and representing valence bond wave functions, overlaps between determinants, valence bond formalism, elementary interactions, structural coefficients and weights, and bridges between molecular orbital and VB theories. Chapters 4, 5, and 6 are concerned with the use of valence bond theory, covering topics such as generating valence bond structures, a discussion of the failures of the theory, and using VB diagrams for chemical reactivity. Chapter 6 is the most useful chapter in this book giving an insightful viewpoint for chemical reactivity and addressing the problem of the origin of reaction barriers.

Chapters 7, 8, and 9 tackle the computational techniques used to implement valence bond theory. These are very useful reference materials for any chemist who wishes to undertake any computational use of such theory for reaction analysis or to gain an understanding of the techniques used by others. In this context, Chapter 10 is a particularly useful guide for any researcher new to this field.

In summary, I certainly feel that this book would be very useful to have in any library. It is particularly suitable as a basis for a teaching course as the questions and answers for each section are an excellent resource.

A. Trewin 